\title{
A wavelet approach for computing nonlinear wave-wave interactions in discrete spectral wave models
}

\author{
G. Uma ${ }^{1}$ - V. Prabhakar ${ }^{1}$ - S. Hariharan ${ }^{1}$
}

Received: 22 May 2015 / Accepted: 15 December 2015 / Published online: 8 January 2016

(C) Springer International Publishing Switzerland 2016

\begin{abstract}
A wavelet-based attempt is made to estimate the nonlinear interactions for wind wave spectra using Haar wavelets. The nonlinear interactions have been synthesized using the orthogonal basis of the Haar wavelets. The analysis of the nonlinear interactions using wavelets provides an easy way of computing the transfer integral in the WebbResio-Tracy's (WRT) method. The one-dimensional and two-dimensional results confirm the applicability of the wavelets to the nonlinear wave-wave interactions and the approach of multi-resolution analysis ensures the convergence and the accuracy.
\end{abstract}

Keywords Nonlinear interaction - Wave model · Haar wavelets · Multi-resolution analysis · Quadruplets · Action density

\section{Introduction}

The energy conservation for wind generated waves in deep water is stated as

$\frac{\mathrm{d} E}{\mathrm{~d} t}=S_{\mathrm{inp}}+S_{\mathrm{nl}}+S_{\mathrm{wcap}}$,

where $E$ denotes the two-dimensional energy spectrum with respect to the wave number $\vec{k}$ at the spatial position and

\author{
V. Prabhakar \\ prabhakar.v@vit.ac.in \\ G. Uma \\ umasathish82@gmail.com \\ S. Hariharan \\ s.hariharan@vit.ac.in \\ 1 Vellore Institute of Technology University, Chennai, India
}

time $t, S_{\text {inp }}$ is the energy input by wind, $S_{\mathrm{nl}}$ is the nonlinear quadruplet wave-wave interactions and $S_{\text {wcap }}$ is the energy dissipation by white capping, see e.g., (Hasselmann 1960) [that gives Eq. (1)]. In the theoretical description of growth and decay of wind waves, nonlinear wave-wave interactions play an important role, which results in a continuous transfer of energy between the components of the wave field. A full solution of $S_{\mathrm{nl}}$ is time consuming because of its complex functional form and therefore it is impractical for implementation in operational wave models (Cavaleri et al. 2007).

Phillips (1960) studied interactions between waves of arbitrary lengths and directions and found that under certain circumstances resonance occurs in the system from third order, resulting in growth of some components at the expense of others. Hasselmann (1962) derived an expression which describes the irreversible energy transfer between four water waves in resonant mode. This expression is known as the Hasselmann's equation or Boltzmann equation. Hasselmann (1963a) simplified the Boltzmann equation and discussed some of its properties. Hasselmann (1963b) evaluated the Boltzmann equation for a Neumann spectrum on deep water and obtained the positive-negative-positive three lobe structure of the transfer rate within the spectrum. Hasselmann and Hasselmann (1985a,b) developed Discrete Interaction Approximation (DIA) method which is computationally efficient. It has several shortcomings which are reported in Van Vledder (2000). Extensions and modifications to the DIA method are done by Hashimoto and Kawaguchi (2001) and Tolman (2004, 2013a, b). Despite its shortcomings, DIA is employed in third generation wave models such as WAM (see Hasselmann et al. 1988). Webb (1978) employed Dirac delta function properties and analytically removed the $\delta$ functions of Hasselmann's equation. Masuda (1980) performed detailed analysis of the kernel function and tested its scheme with different types of spectra. Young and Van 
Vledder (1993) gave a review of the role played by nonlinear wave-wave interactions in operational wave models. Lavrenov (2001) used Gauss-Legendre quadratures to treat the singularities arising from the manipulation of Boltzmann integral. This work was further developed by GagnaireRenou et al. (2010). Benoit (2005) compared the results of nonlinear wave-wave interactions with two exact methods (Webb 1978; Lavrenov 2001) and approximate techniques. Cavaleri et al. (2007) summarized the phenomenon and modelling of nonlinear four wave interactions.

A computationally fast and accurate method being considered currently on the nonlinear problem, focuses on the improvement of the Webb's method. This method called the WRT method is based on the method of Webb (1978) with contributions from Tracy and Resio (1982) and Resio and Perrie (1991). A detailed description of the WRT method can be found in Van Vledder (2006) who suggested several filtering techniques in both radial and directional resolutions to reduce the computational time. In that paper, Trapezoidal rule is applied to the inner integral, whereas the importance of higher-order quadrature methods such as the GaussLegendre quadrature is also indicated. The method has been implemented in various operational wave prediction models such as Wave Watch III (Tolman 1991), SWAN (Booij et al. 1999), CREST (Ardhuin et al. 2001) and PROWAM (Monbaliu et al. 1999).

In the present work, the WRT method is considered for the calculation of the nonlinear source term. Siraj-ul-Islam et al. (2010) and Imran et al. (2011) urge that wavelets are well suited for evaluating the integrals. Further, through numerical examples they proved that Haar wavelets are computationally efficient over the quadrature methods. The aim of this paper is to employ Haar wavelets to the inner integral, which needs the calculation of nodes alone as the weights are constant. Through multi-resolution analysis, the convergence and accuracy of the method is substantiated.

The structure of the paper is as follows: Sect. 2 contains a brief description of the WRT method used for solving the nonlinear source term, and Sect. 3 deals with (i) the basics of multi-resolution analysis, (ii) the application of Haar wavelets to the transfer integral in the WRT method and (iii) a comparative study of the results for the nonlinear source term using the present WRT method with the earlier results of Resio and Perrie (1991) and benchmark results of the WRT method. Finally, the applicability and advantages of the present method is summarized in Sect. 4.

\section{Webb-Resio-Tracy's method}

The nonlinear source term in the wave model (Hasselmann 1962),

$$
\begin{aligned}
\frac{\partial n_{1}}{\partial t}= & \iiint G\left(\overrightarrow{k_{1}}, \overrightarrow{k_{2}}, \overrightarrow{k_{3}}, \overrightarrow{k_{4}}\right) \times \delta\left(\overrightarrow{k_{1}}+\overrightarrow{k_{2}}-\overrightarrow{k_{3}}-\overrightarrow{k_{4}}\right) \\
& \times \delta(W) \times D\left(\overrightarrow{k_{1}}, \overrightarrow{k_{2}}, \overrightarrow{k_{3}}, \overrightarrow{k_{4}}\right) \mathrm{d} \overrightarrow{k_{2}} \mathrm{~d} \overrightarrow{k_{3}} \mathrm{~d} \overrightarrow{k_{4}},
\end{aligned}
$$

describes the rate of change of action density $n_{1}$ at a particular wave number $\overrightarrow{k_{1}}$ due to all the wave resonating quadruplet interactions involving in it. Here, $G$ and $\delta(\cdot)$ denote the coupling coefficients and Dirac delta function, respectively. The term $W$ is defined by $W=\omega_{1}+\omega_{2}-\omega_{3}-\omega_{4}$, where $\omega_{i}$ is the angular frequency corresponding to the $i$ th wave number $k_{i}(i=1, \ldots 4)$ and $n_{i}=n\left(\overrightarrow{k_{i}}\right)$ represent the action density spectrum which is related to the wave number spectrum by $n_{i}=\frac{F\left(\vec{k}_{i}\right)}{\omega_{i}}$. The expression for $G$ can be found in Webb (1978). The density term containing the product of action densities is a cubic expression given by $D\left(\overrightarrow{k_{1}}, \overrightarrow{k_{2}}, \overrightarrow{k_{3}}, \overrightarrow{k_{4}}\right)=\left[n_{1} n_{3}\left(n_{4}-n_{2}\right)+n_{2} n_{4}\left(n_{3}-n_{1}\right)\right]$.

The contribution to the integral in Eq. (2) comes from the set of all three wave number vectors $\left\{\overrightarrow{k_{2}}, \overrightarrow{k_{3}}, \overrightarrow{k_{4}}\right\}$ interacting with $\overrightarrow{k_{1}}$ due to the presence of the delta functions. In other words, this contribution is obtained from the set of all four wave vectors $\left\{\overrightarrow{k_{1}}, \overrightarrow{k_{2}}, \overrightarrow{k_{3}}, \overrightarrow{k_{4}}\right\}$ each satisfying the following resonance conditions

$\overrightarrow{k_{1}}+\overrightarrow{k_{2}}=\overrightarrow{k_{3}}+\overrightarrow{k_{4}}$

and

$\omega_{1}+\omega_{2}=\omega_{3}+\omega_{4}$

The linear dispersion relation relating the angular frequency $\omega$ and the wave number $k$ is expressed as

$\omega^{2}=g k \tanh (k d)$,

where $g$ is the acceleration due to gravity and $d$ is the water depth.

The $\delta$-function over the wave numbers in Eq. (2) is eliminated by integrating over $\overrightarrow{k_{4}}$, to form

$\frac{\partial n_{1}}{\partial t}=\int T\left(\overrightarrow{k_{1}}, \overrightarrow{k_{3}}\right) d \overrightarrow{k_{3}}$

with the transfer integral $T\left(\overrightarrow{k_{1}}, \overrightarrow{k_{3}}\right)$ given by

$T\left(\overrightarrow{k_{1}}, \overrightarrow{k_{3}}\right)=\int G \times D\left(\overrightarrow{k_{1}}, \overrightarrow{k_{2}}, \overrightarrow{k_{3}}, \overrightarrow{k_{4}}\right) \times \delta(W) \mathrm{d} \overrightarrow{k_{2}}$.

In the present work, the entire domain is considered and no filtering technique is used.

An important step in the WRT method is to find the wave resonating quadruplets $\left\{\overrightarrow{k_{1}}, \overrightarrow{k_{2}}, \overrightarrow{k_{3}}, \overrightarrow{k_{4}}\right\}$. This can be 
achieved by fixing two wave number vectors. Thus, for input vectors $\overrightarrow{k_{1}}$ and $\overrightarrow{k_{3}}$, the locus of $\overrightarrow{k_{2}}$ traces out an egg-shaped closed curve. For the case when the magnitudes of the input vectors are the same, the locus of $\overrightarrow{k_{2}}$ is a straight line. The locus of $\overrightarrow{k_{4}}$ can then be constructed using Eq. (3).

Further, $\overrightarrow{k_{2}}$ is segregated into tangential-normal coordinate $\operatorname{system}(\vec{s}, \vec{n})$ as $\overrightarrow{k_{2, s}}$ and $\overrightarrow{k_{2, n}}$. Using the properties of the Dirac delta function and integrating along the normal component $\overrightarrow{k_{2, n}}$, the nonlinear transfer integral in Eq. (6b) reduces to a line integral of the form

$T\left(\overrightarrow{k_{1}}, \overrightarrow{k_{3}}\right)=\oint G \times D\left(\overrightarrow{k_{1}}, \overrightarrow{k_{2}}, \overrightarrow{k_{3}}, \overrightarrow{k_{4}}\right) \times J \times \mathrm{d} s$.

Here $J=\left|\frac{\mathrm{d} W}{\mathrm{~d} n}\right|^{-1}$ is the normal derivative term or Jacobian.

Now, Eq. (6a) is written in terms of polar coordinates as

$\frac{\partial n\left(k_{1}, \theta\right)}{\partial t}=\iint T\left(\overrightarrow{k_{1}}, \overrightarrow{k_{3}}\right) k_{3} \mathrm{~d} \theta_{3} \mathrm{~d} k_{3}$.

Following Tracy and Resio (1982), the double integral in Eq. (8) is evaluated. Having computed $\frac{\partial n(k, \theta)}{\partial t}, S_{\mathrm{nl}}(f, \theta)$ is retrieved using the relation $S_{\mathrm{nl}}(f, \theta)=\frac{4 \pi \omega^{4}}{g^{2}} \frac{\partial n(k, \theta)}{\partial t}$. The one-dimensional nonlinear source term $S_{\mathrm{nl}}(\stackrel{f}{f})$ is determined by integrating $S_{\mathrm{nl}}(f, \theta)$ with respect to $\theta$.

\section{Multi-resolution analysis and Haar wavelets}

The application of wavelets to the nonlinear wave-wave interactions starts with the approximation of the integrand in the transfer integral using Haar wavelets through multiresolution analysis and ends up with the integration scheme.

\subsection{Multi-resolution analysis or multi-level representation of function}

The aim of multi-resolution analysis is to develop representation of a function $f(x)$ at various levels of resolution. To this end, we seek to expand $f(x) \in L^{2}(R)$ in terms of basis functions called scaling function $\phi(x)$ and the wavelet function $\psi(x)$ which can be scaled to give the multiple resolution of the function.

A multi-resolution analysis (MRA) of the set of square integrable functions denoted by $L^{2}(R)$, equipped with the standard inner product $(\cdot, \cdot)$, is a chain of closed subspaces indexed by all integers

$$
\ldots V_{-1} \subset V_{0} \subset V_{1} \ldots
$$

such that

(i) $\bigcup V_{n}=L^{2}(R)$

(ii) $\bigcap_{n}^{n} V_{n}=\{0\}$

(iii) $\stackrel{n}{f}(\cdot) \in V_{n} \Leftrightarrow f(2 \cdot) \in V_{n+1}$

(iv) Let $\phi(\cdot)$ be a scaling function such that $\{\phi(\cdot-k): k \in Z\}$ constitutes a complete orthonormal basis of $V_{0}$.

To obtain an MRA, it suffices to construct the scaling function $\phi(\cdot)$. The entire space chain can then be reconstructed from $\phi(\cdot)$ according to (iii) and (iv). Since $V_{0} \subset V_{1}$ and from (iii) and (iv), it is easy to see that $\phi(\cdot)$ must be a linear combination of $\phi(\cdot-k): k \in Z$, leading to the two scale relation

$\phi(\cdot)=2 \sum_{k \in Z} h_{k} \phi(2 \cdot-k)$

for a suitable set of coefficients $\left(\ldots, h_{-1}, h_{0}, h_{1}, \ldots\right)$.

Let $W_{0}$ denote the orthogonal complement of $V_{0}$ in $V_{1}$. A function $\psi(\cdot)$ whose integer translates $\{\psi(\cdot-k): k \in Z\}$ constitutes an orthonormal basis of $W_{0}$ is called a wavelet. This wavelet function $\psi(\cdot)$ satisfies the two scale relation

$\psi(\cdot)=2 \sum_{k \in Z} g_{k} \psi(2 x-k)$,

for a suitable set of coefficients $\left(. ., g_{-1}, g_{0}, g_{1}, \ldots\right)$. From (i)-(iv) it is clear that

$\phi_{j, k}(\cdot)=2^{j / 2} \phi\left(2^{j} \cdot-k ; j, k \in Z\right)$.

and

$\psi_{j, k}(\cdot)=2^{j / 2} \psi\left(2^{j} \cdot-k ; j, k \in Z\right)$.

form an orthonormal bases of $L^{2}(R)$, where $j$ and $k$ are the scaling and translating parameters. For the well-known Haar wavelet, the scaling and wavelet functions are given by

$\phi(x)= \begin{cases}1, & 0 \leq x<1 \\ 0, & \text { otherwise }\end{cases}$

$\psi(x)= \begin{cases}1, & 0 \leq x<\frac{1}{2} \\ -1, & \frac{1}{2} \leq x<1\end{cases}$

Figure 1 shows the relationship between scaling and wavelet functions at different levels, i.e., $V_{n}=V_{n-1} \oplus W_{n-1}$.

For any function $L^{2}(R)$, define $P_{J}: L^{2}(R) \longrightarrow V_{J}$ to be the projection of $f$ onto the resolution space $V_{J}$ by 


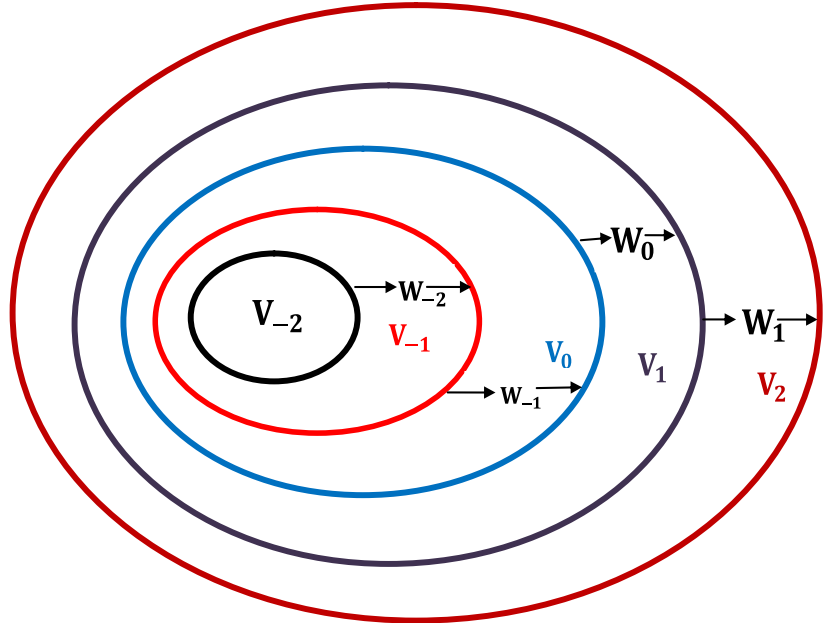

Fig. 1 Relation between $V_{j}$ and $W_{j}$

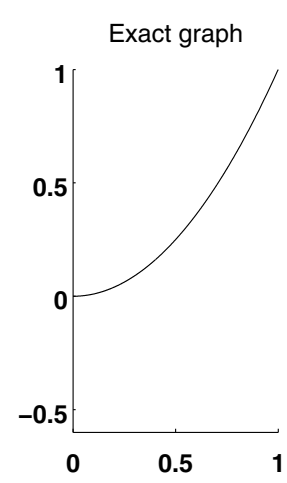

(a) $\mathrm{v}_{0}$

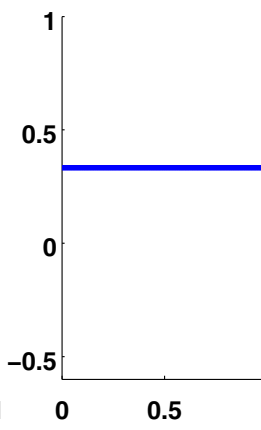

(b)

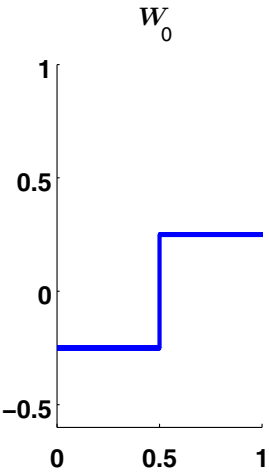

(c)

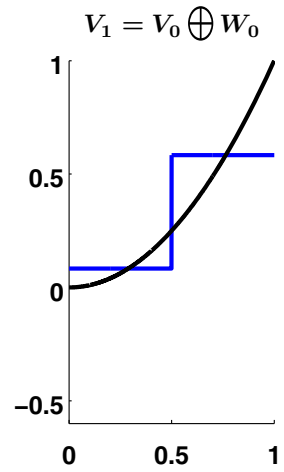

(d)

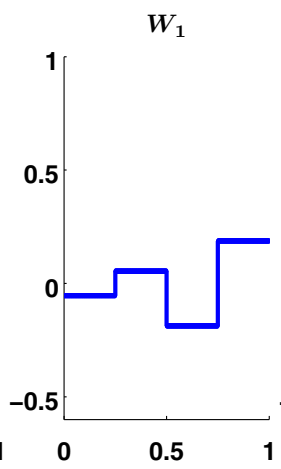

(e)

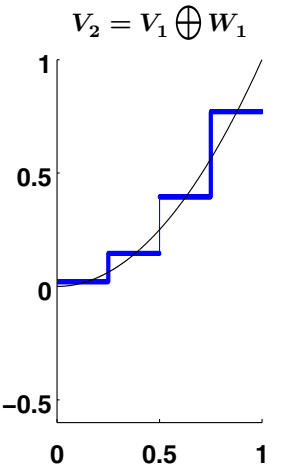

(f)
Fig. 2 MRA for $x^{2}$

$$
\begin{aligned}
f(x)= & \sum_{k \in Z} c_{0, k}(f) \phi_{0, k}(x) \\
& +\sum_{0 \leq j \leq J} \sum_{\substack{0 \leq k \leq 2^{j}-1 \\
k \in Z}} d_{j, k}(f) \psi_{j, k}(x),
\end{aligned}
$$

where

$c_{0, k}=\int f(x) \phi(x-k) \mathrm{d} x$

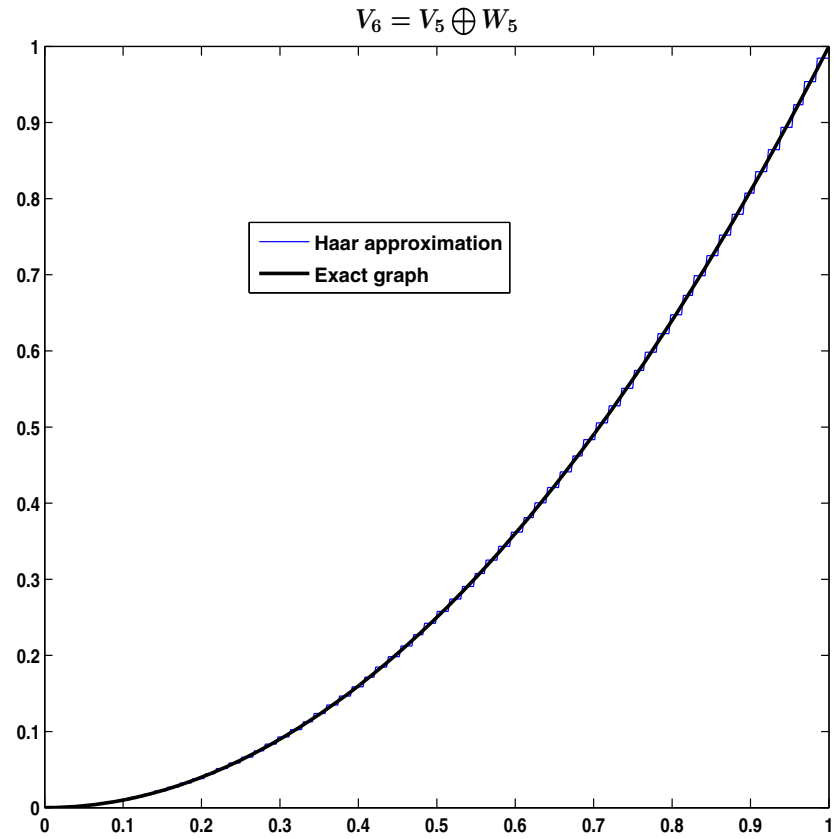

Fig. 3 MRA for $x^{2}$ at $J=5$

and

$d_{j, k}=\int f(x) \psi_{j, k}(x) \mathrm{d} x$.

The parameter $J$ denotes the maximum level of resolution of Haar wavelets to represent the function $f(x)$. The analysis of the function can be done using the scaling coefficients $c_{0, k}$ and the wavelet coefficients $d_{j, k}$. These coefficients are also called the average and detailed coefficients of the corresponding function. Consider

$f(x)= \begin{cases}x^{2}, & 0 \leq x<1 \\ 0, & \text { otherwise }\end{cases}$

In order to approximate $f(x)$ at the resolution level $J=$ 1 , the following coefficients have to be calculated using Eqs. (10) and (11) as follows:

$c_{0,0}=\int_{0}^{1} x^{2} \phi(x) \mathrm{d} x=\frac{1}{3}$,

$d_{0,0}=\int_{0}^{1} x^{2} \psi_{0,0}(x) \mathrm{d} x=\int_{0}^{0.5} x^{2} \mathrm{~d} x-\int_{0.5}^{1} x^{2} \mathrm{~d} x=\frac{-1}{4}$,

$d_{1,0}=\int_{0}^{1} x^{2} \psi_{1,0}(x) \mathrm{d} x=\int_{0}^{0.25} x^{2} \mathrm{~d} x-\int_{0.25}^{0.5} x^{2} \mathrm{~d} x=-\frac{\sqrt{2}}{32}$, 

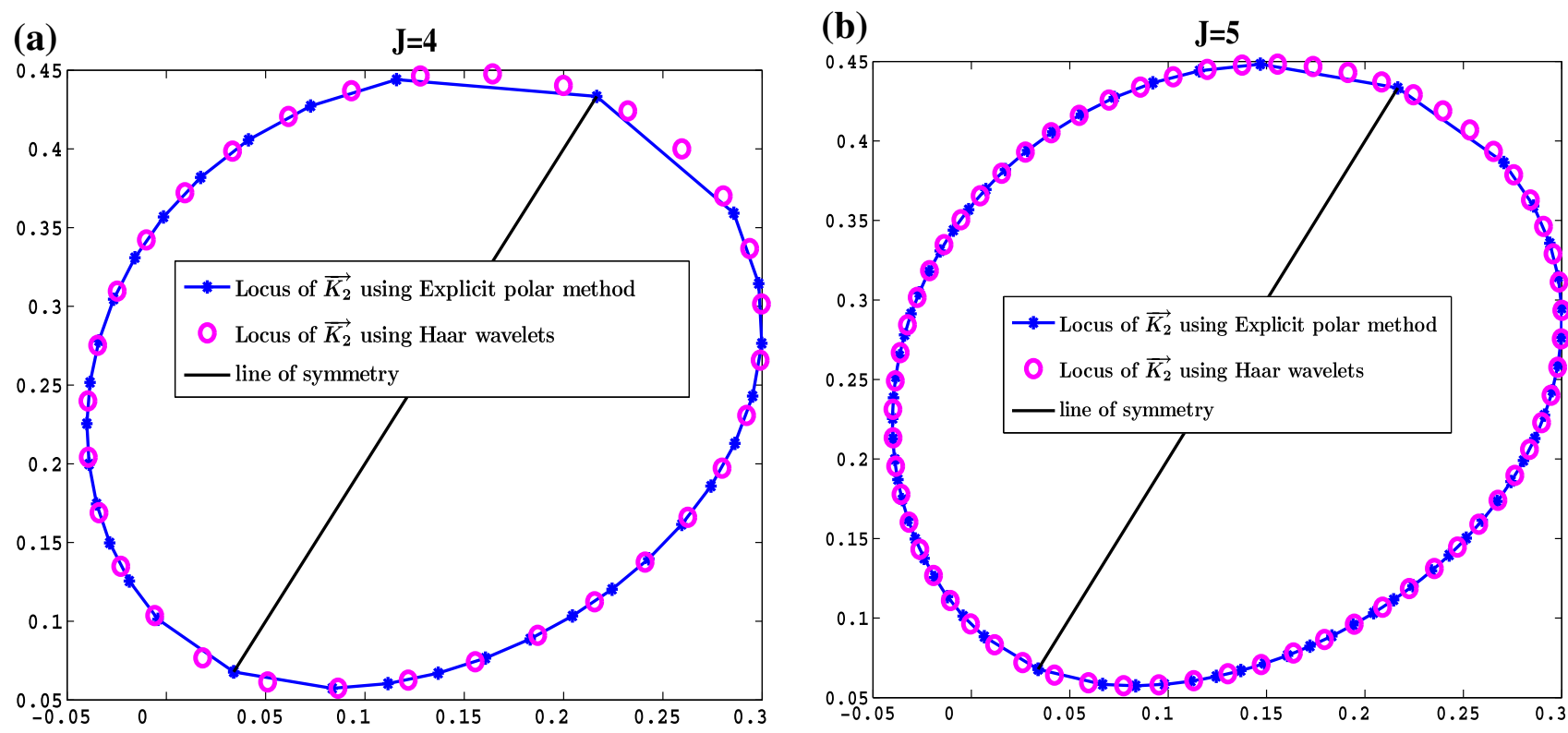

(c) $\mathrm{J}=4$
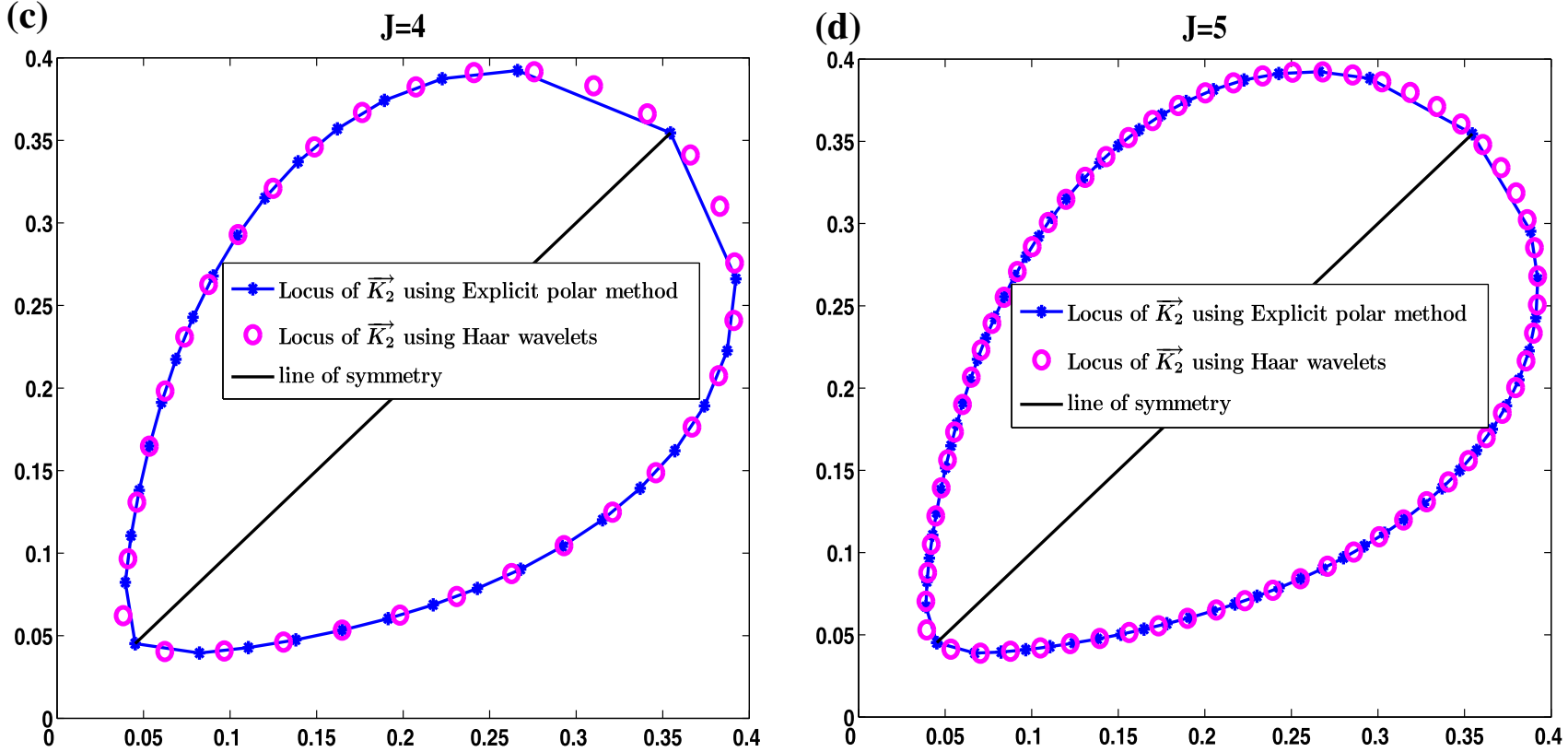

Fig. 4 Approximation of locus results of $\overrightarrow{k_{2}}$ for both deep and finite depths using Haar wavelets at different levels of resolution

and

$d_{1,1}=\int_{0}^{1} x^{2} \psi_{1,1}(x) \mathrm{d} x=\int_{0.5}^{0.75} x^{2} \mathrm{~d} x-\int_{0.75}^{1} x^{2} \mathrm{~d} x=-\frac{3 \sqrt{2}}{32}$.

Using Eq. (9), $f(x) \approx\left[\frac{1}{3} \phi_{0,0}(x)\right]+\left[\frac{-1}{4} \psi_{0,0}(x)\right]+$ $\left[-\frac{\sqrt{2}}{32} \psi_{1,0}(x)+\frac{-3 \sqrt{2}}{32} \psi_{1,1}(x)\right]$.

The function approximation is shown in Fig. 2f. As the level increases, the function approximation converges towards the function $f$ which can be seen from Fig. 3 with $J=5$.
For a detailed introduction to wavelet theory, refer to Strang and Nguyen (1996) and Hernandez and Weiss (1996). The evaluation of the definite integrals using Haar wavelets can be carried out by

$\int_{a}^{b} f(x) \mathrm{d} x=\frac{b-a}{2^{J+1}} \sum_{q=1}^{2^{J+1}} f\left(x_{q}\right)$

where $x_{q}=a+\frac{(b-a)(q-0.5)}{2^{J+1}}$, with $J$ representing the maximum level of resolution of Haar wavelets. More details can be found in Siraj-ul-Islam et al. (2010) and Imran et al. (2011). 


\subsection{Procedure for approximating the locus curve using Haar wavelets}

Let $\Omega$ be the locus of $\overrightarrow{k_{2}}$ in the Cartesian plane. Let $\Omega$ be approximated by a parametric cubic spline curve $\Gamma$ : $(x(s), y(s))$, where $s$ is the normalized cumulative chord length and $x(s)$ and $y(s)$ are the cubic splines (Hanna et al. 1986). The steps involved in this procedure are described below.

Step 1 Obtain an ordered set of points $\left\{x_{i}, y_{i}\right\}_{i=0}^{n-1}$ on the locus of $\overrightarrow{k_{2}}$, using a standard polar method such as the explicit polar method presented in Van Vledder (2000), with starting point on the symmetry axis.

The standard polar method consists of obtaining first the end radial points (or minimum and maximum radial values) of $\overrightarrow{k_{2}}$ on the symmetry axis and then obtaining the points on either side of the locus for which the radial values of $\overrightarrow{k_{2}}$ vary between this maximum and minimum values. In this method, $n$ number of points on the symmetry axis are chosen to give $2(n-1)$ points on the locus. Thus, the number of points on the locus of $\overrightarrow{k_{2}}$ is always even.

Step 2 Obtain the cubic splines $x(s)$ and $y(s)$ (Gerald and Wheatly 2008) from the points of $\Omega$ as follows.

Let $s_{i}$ denote the value of the normalized cumulative chord length at the $i$ th point on the locus $\Omega$. For the data $\left\{s_{i}, x_{i}\right\}_{i=0}^{n-1}$ and $\left\{s_{i}, y_{i}\right\}_{i=0}^{n-1}$, obtain the cubic splines $x(s)$ and $y(s)$ using not-a-knot end conditions, respectively. For a closed locus, the parameter $s$ varies from 0 to 1 .

Step 3 By considering the discrete values of $s$ to be the nodes of the Haar wavelets and using the cubic spline approximants $x(s)$ and $y(s)$ for the locus points, one can obtain a new set of points on the locus of $\overrightarrow{k_{2}}$. Since the nodes of the Haar wavelets are equally spaced, the locus points are uniformly distributed.

The top panel of Fig. 4 shows the distribution of 32 and 64 points on the locus of $\overrightarrow{k_{2}}$ for an input pair of wave number vectors $\overrightarrow{k_{1}}=(0.15,0)$ and $\overrightarrow{k_{3}}=(0.2,0.1)$. The locus curves are approximated using Haar wavelets, corresponding to the fourth and fifth levels of resolution. From this, it was found that the new set of points are equispaced with the spacing being $0.07,0.036$ and 0.018 approximately, corresponding to $J=3,4$ and 5, respectively. The bottom panel of Fig. 4 shows the distribution of 32 and 64 points on the locus of $\overrightarrow{k_{2}}$ for an input pair of wave number vectors $\overrightarrow{k_{1}}=(0.1,0)$ and $\overrightarrow{k_{3}}=(0.15,0.05)$ with depth $1.5 \mathrm{~m}$. The locus curves are approximated using Haar wavelets, corresponding to the fourth and fifth levels of resolution. Here, it was found that the new set of points are equispaced with the spacing being $0.07,0.035$ and 0.017 approximately, corresponding to the levels 3,4 and 5, respectively.

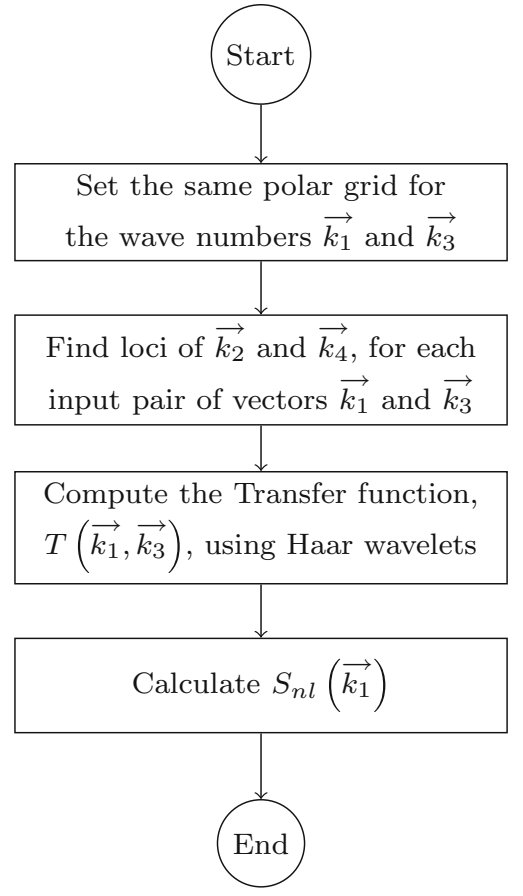

Fig. 5 Flow chart showing the computation of nonlinear transfer

Application of Haar wavelets to Eq. (7) yields,

$T\left(\overrightarrow{k_{1}}, \overrightarrow{k_{3}}\right)=\frac{L}{2^{J+1}} \sum_{k=1}^{2^{J+1}} G\left(s_{k}\right) \times D\left(s_{k}\right) \times J\left(s_{k}\right)$,

where $s_{k}$ are the Haar nodes along the locus of $\overrightarrow{k_{2}}, L$ is the length of the closed curve and $J$ is the maximum level of resolution. For the lower limit of line integration, $(a)$ is always 0 and the upper limit $(b)$ is $L$. Subsequently, Eq. (8) is evaluated.

Figure 5 depicts the flow chart and further explanations about the computation of nonlinear transfer.

To compute the nonlinear transfer, a polar grid with 60 different wave numbers and 36 different angles have been considered. In these calculations, the directional resolution is $10^{\circ}$, ranging from $0^{\circ}$ to $360^{\circ}$, and the frequencies are geometrically spaced. The input spectrum is chosen to be the JONSWAP spectrum with -5 tail, and the shape parameters are $\alpha=0.01, f_{p}=0.3 \mathrm{~Hz}$ and $\sigma=\left\{\begin{array}{ll}0.07, & f<f_{p} \\ 0.09, & f \geq f_{p} .\end{array}\right.$. The two-dimensional input frequency-direction spectra are shown in Fig. 6.

Figure 7 shows the comparison of 1-D nonlinear transfer results of $S_{\mathrm{nl}}(f)$ for the PM spectrum, JONSWAP spectra with peak enhancement factor $\gamma=3.3$ and $\gamma=7$, respectively, using the present WRT method with the ear- 
Fig. 6 Input

frequency-directional distribution $E(f, \theta)$ for different peak enhancement factors: a $\gamma=1, \mathbf{b} \gamma=3.3$ and $\mathbf{c} \gamma=7$
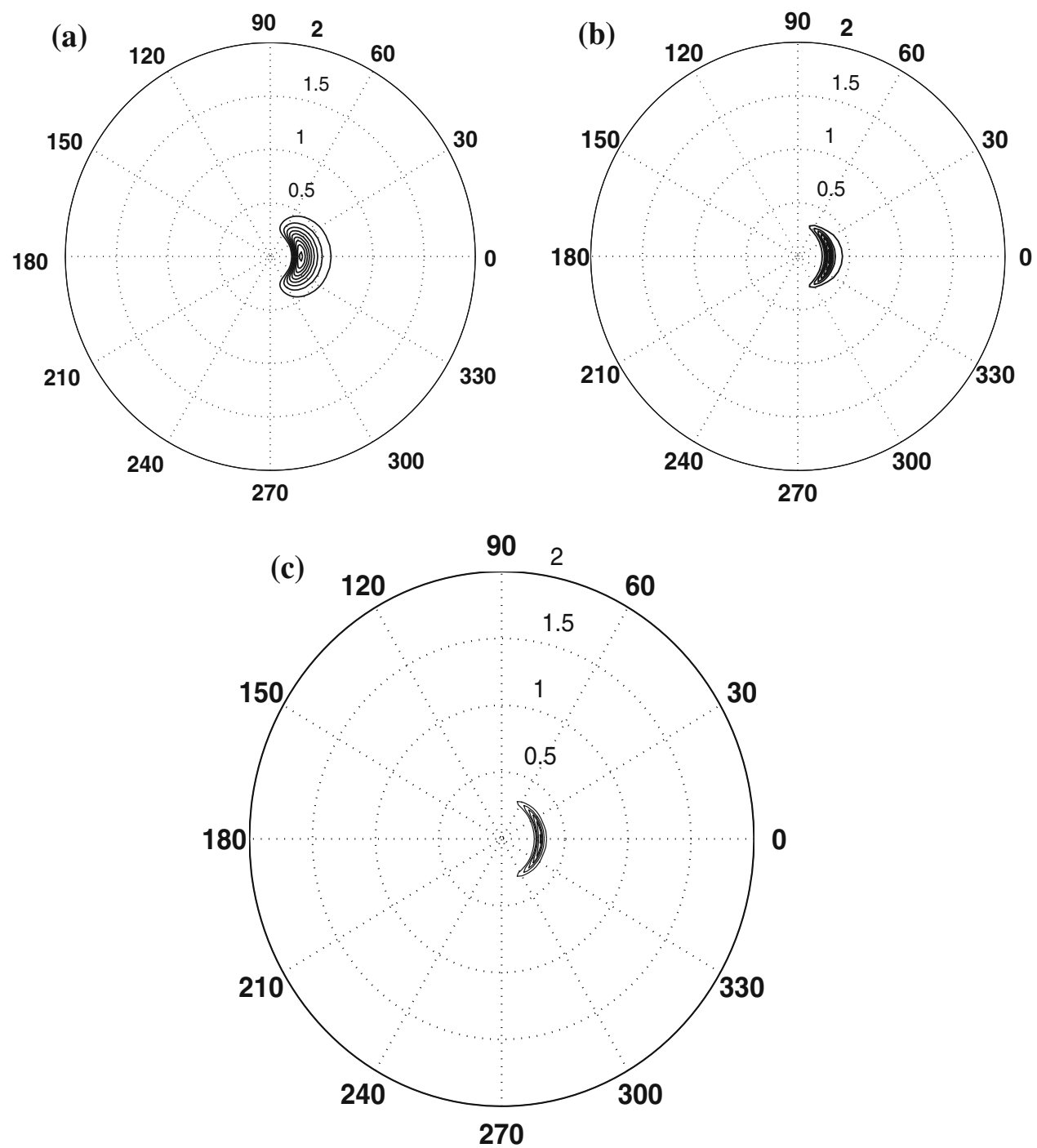

lier results. In the present method, $J$ is chosen to be 4 for the PM spectrum, and 5 for the other two spectra. In the earlier results of Resio and Perrie (1991), sector grid ranging from $-120^{\circ}$ to $120^{\circ}$ was considered for integration, whereas the present method and the benchmark of the WRT results use circular grid. It is clear from Fig. 7 that the present results are comparable and in good agreement with earlier results.

Figure 8 depicts the 2-D nonlinear transfer results obtained using the present method for the same input spectra and the level of resolution $J$ considered in Fig. 6 .

The effect of the directional resolution on the directional transfer rate $S_{\mathrm{nl}}(\theta)$, for the varying values of $\gamma$ are shown in Fig. 9. Here, $\theta$ ranges from $0^{\circ}$ to $360^{\circ}$ with $10^{\circ}$ spacing. As $\gamma$ increases, the two positive lobes and the negative lobe approach a narrow distribution.

Better accuracy of the nonlinear transfer results can be achieved by increasing the maximum level of resolution $J$. Figure 10 shows the convergence of the 1-D nonlinear transfer $S_{\mathrm{nl}}(f)$ of the present method for the input PM spectrum with increasing values of $J$. The adaptation of wavelets have been explored by varying the number of locus points as 8 , 16, 32 and 64, which fixes the resolution levels as 2, 3, 4 and 5 , respectively. A detailed study has to be made regarding the performance of the present method (accuracy vs computational time) and will be considered in our future work.

\section{Conclusions}

In this paper, the potential of Haar wavelets for the nonlinear wave-wave interactions in the WRT method is examined at different resolution levels. This is achieved by applying MRA using Haar wavelets for the transfer integral. The advantage of applying Haar wavelets is that it involves constant weights and equal spacing of the nodes. The adaptation of wavelets have been explored by varying the number of locus points which fixes the resolution level $J$. Variations of nonlinear transfer with respect to both frequency and direc- 

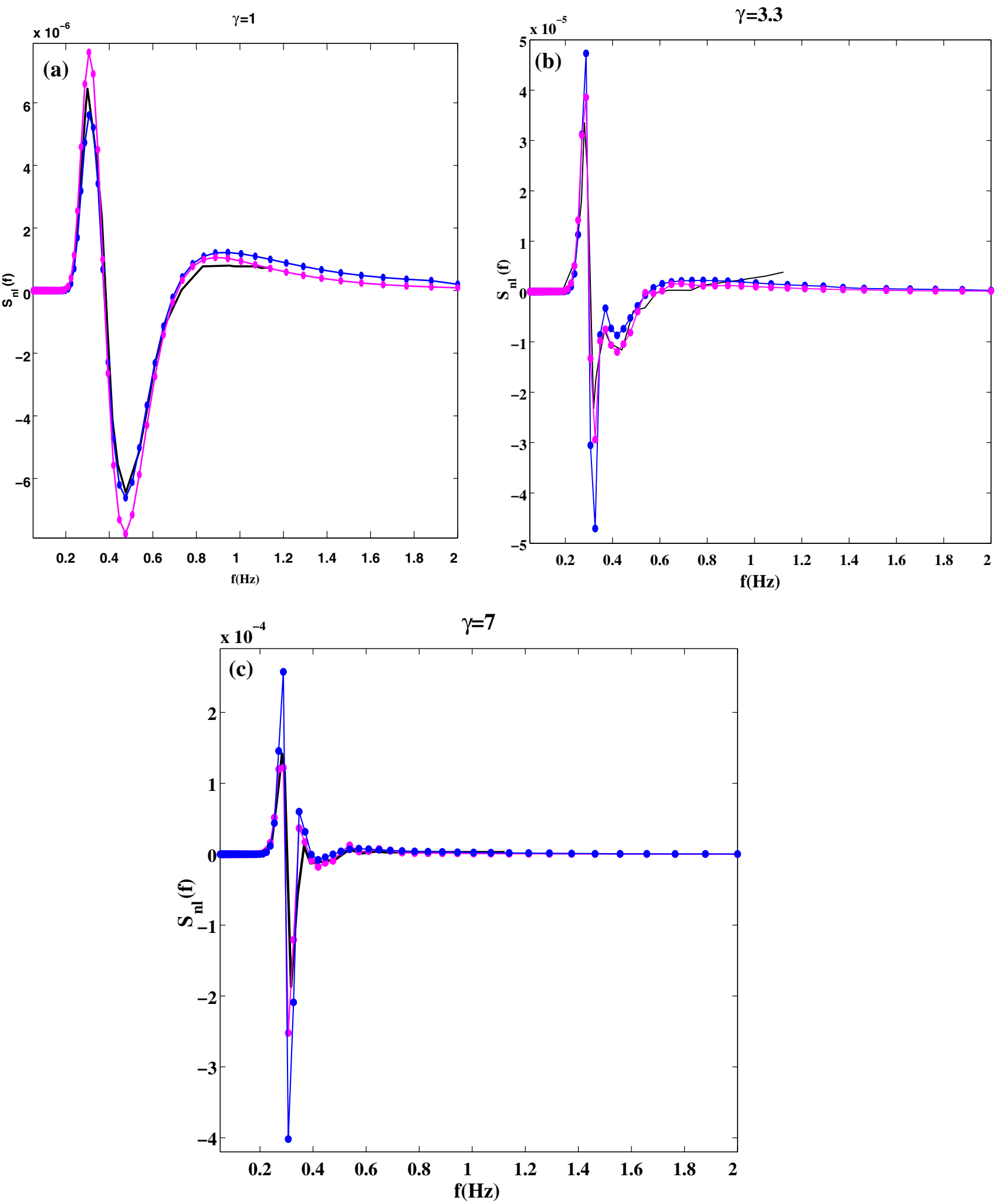

Fig. 7 Comparison of 1-D nonlinear transfer results $S_{\mathrm{nl}}(f)$, using the present WRT method (blue lines with dots) with benchmark of the WRT results (pink line with dots) and the WRT results taken from Resio and
Perrie (1991) (straight line) for case 13, case 2 and case 15 of Hasselmann and Hasselmann (1981) 


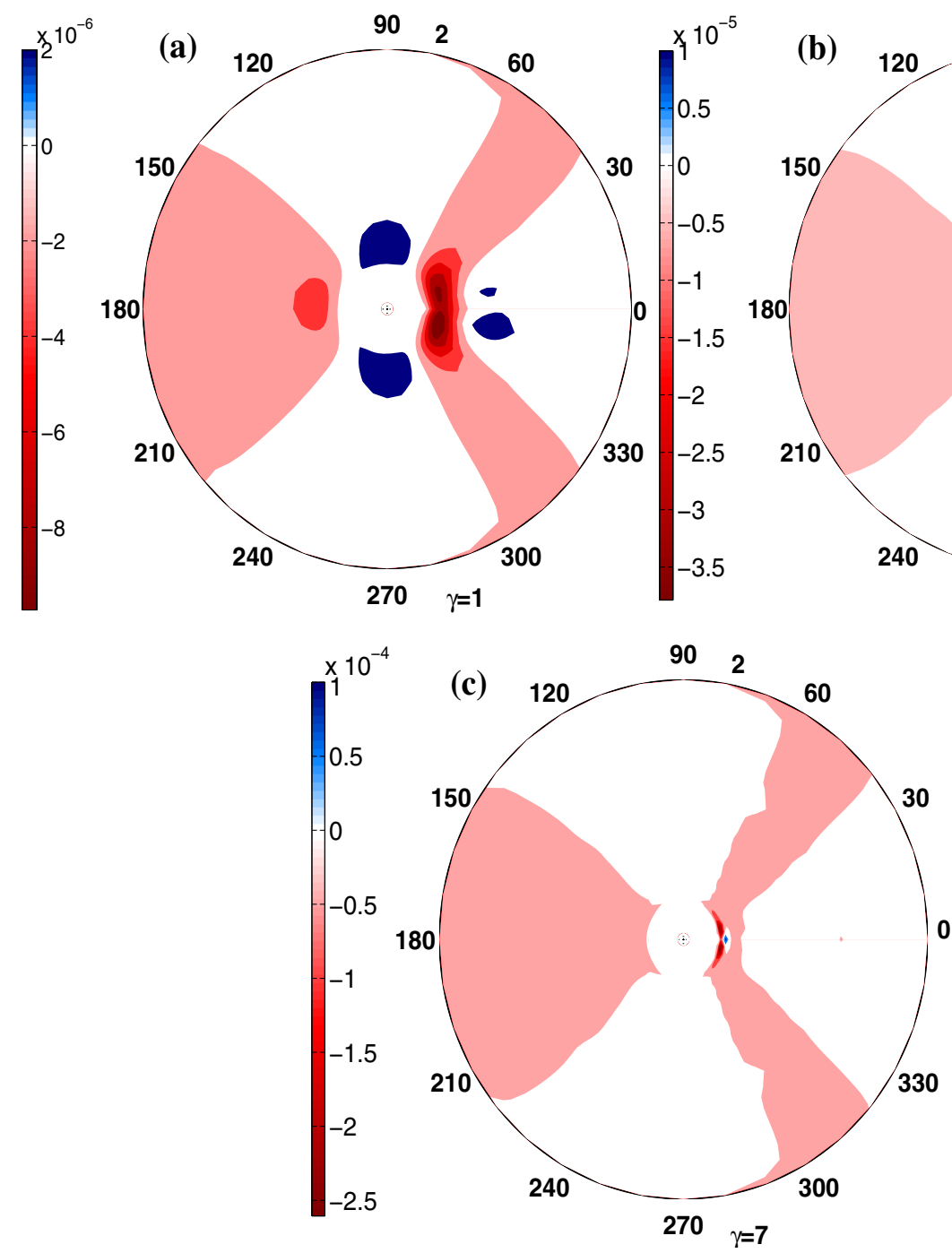

Fig. 8 Two-dimensional nonlinear transfer results $S_{\mathrm{nl}}(f, \theta)$

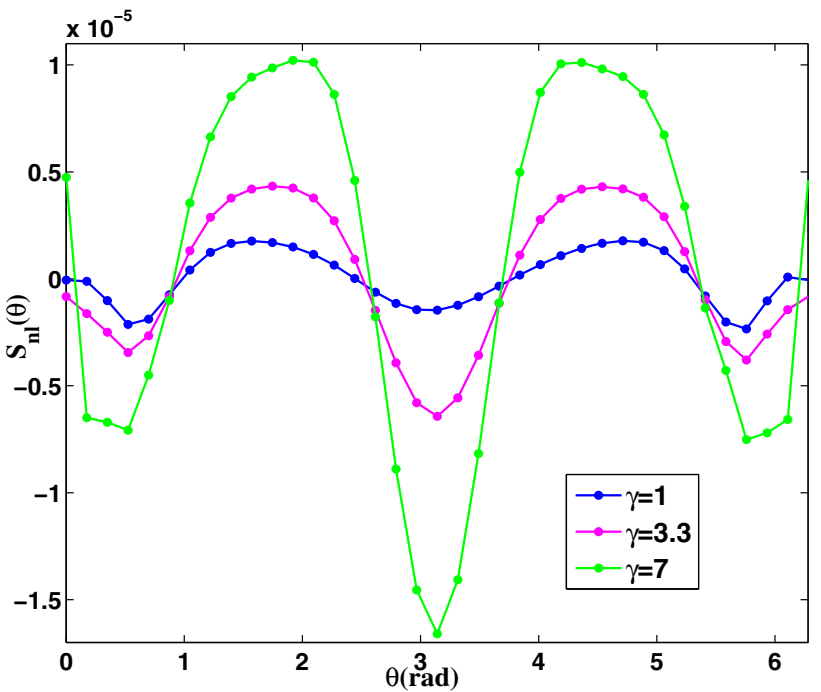

Fig. 9 Frequency-integrated nonlinear transfer results $S_{\mathrm{nl}}(\theta)$ as a function of direction $\theta$ for 3 values of peakedness parameter

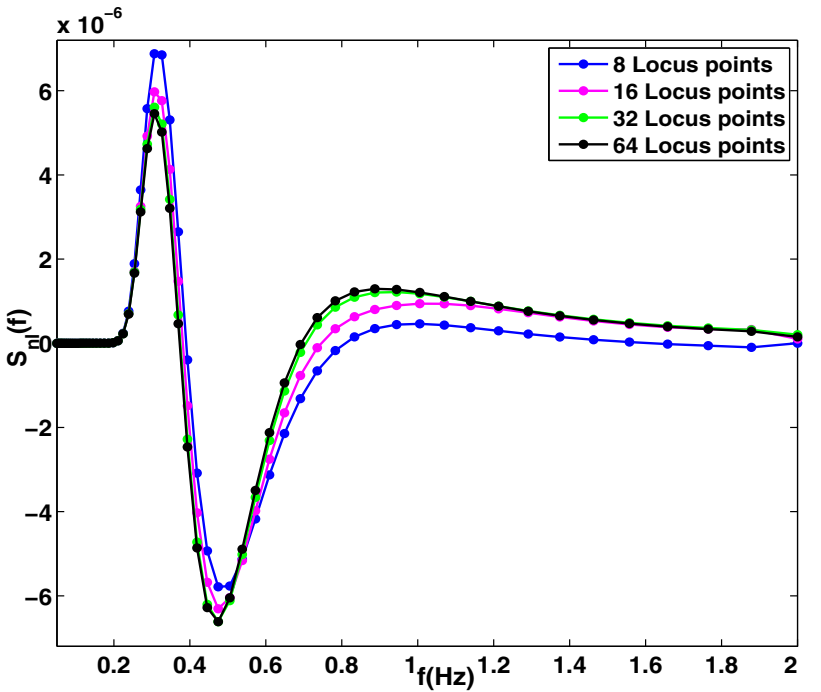

Fig. $101-D$ nonlinear transfer results for $\gamma=1$ with varying points on the locus 
tion are discussed. The one-dimensional nonlinear results obtained through Haar wavelets are found to be comparable with earlier results. The study of applying other wavelets to the nonlinear wave-wave interactions through the WRT method will be considered as a continuation of the present work.

Acknowledgments The second author (VP) expresses his gratitude to CSIR, India, for their funding support to this research project through the CSIR Sanction No. 22 (0562)/11/EMR-II. The first author (GU) is grateful to CSIR for providing financial support through Senior Research Fellowship. We thank Dr. Gerbrant Van Vledder, for his fruitful suggestions and discussions in understanding the model. Further, we gratefully acknowledge him, for providing us the benchmark of the WRT results. We also thank Dr. William Perrie, Bedforth Institute of Oceanography, for his valuable comments, made in early stage of developing the model. The authors thank the editors and anonymous referees for their valuable comments and suggestions which led to an improvement of our original manuscript.

\section{References}

Ardhuin F, Herbers THC, O'Reilly WC (2001) A hybrid Eulerian Lagrangian model for spectral wave evolution with application to bottom friction on the continental shelf. J Phys Oceanogr 31:14981516

Benoit M (2005) Evaluation of methods to compute the nonlinear quadruplet interactions for deep-water wave spectra. In: Proceedings of the 5th ASCE international symposium on ocean waves, measurements and analysis, Madrid

Booij N, Ris RC, Holthuijsen LH (1999) A third generation wave model for coastal regions I. Model description and validation. J Geophys Res 104(C4):7649-7666

Cavaleri L, Alves JHGM, Ardhuin F, Babanin A, Banner M, Belibassakis K, Benoit M, Donelan M, Groeneweg J, Herbers THC, Hwang P, Janssen PAEM, Janssen T, Lavrenov IV, Magne R, Monbaliu J, Onorato M, Polnikov V, Resio D, Rogers WE, Sheremet A, McKee Smith J, Tolman HL, Van Vledder G, Wolf J, Young I; the WISE group (2007) Wave modelling - the state of the art. Prog Oceanogr 75:603-674

Gagnaire-Renou E, Benoit M, Forget P (2010) Ocean wave spectrum properties as derived from quasi-exact computations of nonlinear wave-wave interactions. J Geophys Res 115:C12058

Gerald F, Wheatly O (2008) Applied numerical analysis. Pearson, New Delhi

Hanna MS, Evans DG, Schweitzer PN (1986) On the approximation of plane curves by parametric cubic splines. BIT 26:217-232

Hashimoto N, Kawaguchi K (2001) Extension and modification of the discrete interaction approximation (DIA) for computing nonlinear energy transfer of gravity wave spectra. In: Proceedings of the 4th ASCE international symposium on ocean waves, measurement and analysis, San Francisco, pp 530-539

Hasselmann K (1960) Grundgleichungen der seegangsvoraussage. Schiffstechnik 7:191-195

Hasselmann K (1962) On the nonlinear energy transfer in a gravitywave spectrum, Part 1. General theory. J Fluid Mech 12:481-500

Hasselmann K (1963a) On the nonlinear energy transfer in a gravity wave spectrum, Part 2. Conservation theorems; wave-particle analogy; irreversibility. J Fluid Mech 15:273-281

Hasselmann K (1963b) On the nonlinear energy transfer in a gravity wave spectrum, Part 3. Evaluation of energy flux and swell-sea interaction for a Neumann spectrum. J Fluid Mech 15:385-398
Hasselmann K, Hasselmann S (1981) A symmetrical method of computing the non-linear transfer in a gravity wave spectrum. Hambg Geophys (Einzelschriften, Heft) 52:138

Hasselmann S, Hasselmann K (1985a) Computations and parameterizations of the nonlinear energy transfer in a gravity-wave spectrum. Part 1. A new method for efficient computations of the exact nonlinear transfer integral. J Phys Oceanogr 15:1369-1377

Hasselmann S, Hasselmann K (1985b) Computations and parameterizations of the nonlinear energy transfer in a gravity-wave spectrum. Part 2. Parameterizations of the nonlinear energy transfer for application in wave models. J Phys Oceanogr 15:1378-1391

Hernandez E, Weiss G (1996) A first course on wavelets. CRC Press, New York

Hasselmann S, Hasselmann K, Komen GK, Janssen P, Ewing JA, Cardone V (1988) The WAM model — a third generation ocean wave prediction model. J Phys Oceanogr 18:1775-1810

Imran A, Siraj-ul-islam, Khan W (2011) Quadrature rules for numerical integration based on Haar wavelets and hybrid functions. Comput Math Appl 61:2770-2781

Lavrenov IV (2001) Effect of wind wave parameter fluctuation on the nonlinear spectrum evolution. J Phys Oceanogr 31:861-873

Masuda A (1980) Nonlinear energy transfer between wind waves. J Phys Oceanogr 10:2082-2093

Monbaliu J, Hargreaves JC, Carretero JC, Gerritsen H, Flather R (1999) Wave modelling in the PROMISE project. Coast Eng 37:379-407

Phillips OM (1960) On the dynamics of unsteady gravity waves of finite amplitude. Part 1, The elementary interactions. J Fluid Mech 9:193-217

Resio DT, Perrie W (1991) A numerical study of nonlinear energy fluxes due to wave-wind interactions, Part I. Methodology and basic results. J Fluid Mech 223:603-629

Siraj-ul-Islam, Imran A, Haq F (2010) A comparative study of numerical integration based on Haar wavelets and hybrid functions. Comput Math Appl 59:2026-2036

Strang G, Nguyen T (1996) Wavelets and filter banks, Wesley. Cambridge Press, Cambridge

Tolman HL (1991) A third-generation model for wind waves on slowly varying, unsteady and inhomogeneous depths and currents. J Phy Oceanogr 21:782-797

Tolman HL (2004) Inverse modeling of discrete interaction approximations for nonlinear interactions in wind waves. Ocean Model 6:405-422

Tolman HL (2013a) A generalized multiple discrete interaction approximation for resonant four-wave interactions in wind wave models. Ocean Model 70:11-24

Tolman HL (2013b) Holistic genetic optimization of a generalised multiple discrete interaction approximation for wind waves. Ocean Model 70:25-37

Tracy N, Resio DT (1982) Theory and calculation of the nonlinear energy transfer between sea waves in deep water. In: WIS report 11. US Army Engineer Waterways Experiment Station, Vicksburg

Van Vledder GPh (2000) Improved method for obtaining the integration space for the computation of nonlinear quadruplet wave-wave interactions. In: Proceedings of the 6th international workshop on wave hindcasting and forecasting, Monterey

Van Vledder GPh (2006) The WRT method for the computation of non-linear four-wave interactions in discrete spectral wave models. Coast Eng 53:223-242

Webb DJ (1978) Non-linear transfers between sea waves. Deep Sea Res 25:279-298

Young IR, Van Vledder GPh (1993) A review of the central role of nonlinear interactions in wind-wave evolution. Philos Trans R Soc Lond A 342:505-524 